\title{
The Application of Meta-Analysis in Econometric
}

\author{
Fen Li \\ School of Mathematical Sciences, Tongji University, Shanghai, 200092, China
}

Keywords: meta-analysis; meta-regression; economy; econometrics.

\begin{abstract}
Meta-analysis can enhance the statistical efficiency of data processing in academic research, effectively save the cost of scientific research and improve the strength of argumentation. To be able to the in-depth and detailed analysis of the differences of individual research results makes the analysis conclusions in many similar research problems more accurate and consistent in academic research. It can solve some problems that cannot be solved by the original independent research institutes, and can lead to new ideas and new propositions. More literature of the same kind can be processed according to the scientific standard procedure and method, making the result of the review more objective and close to reality.
\end{abstract}

\section{Introduction}

In the study of economics, given the same economic problems or the same assumptions tend to be at the same time (or have) many economists to study independently, however, because of the difference of the academic background and analysis of the selected factors composed of angles and methods, making the results of the studies often inconsistent and even contrary[1]. One way to deal with this situation is to make a quantitative analysis combining the results of these independent studies to some extent. Literature review is a common way of comprehensive analysis, but the traditional literature review we used is partial, subjectivity, and the lack of quantitative accuracy. What is more, some reviewers tend to choose only information that supported his arguments and further produce different review opinions of the embarrassing situation, which may make enormous bias.

In recent years, the rapid development of meta-analysis in the academic world is the systematic integration of multiple independent research results conducted by different researchers under the same research topic, the analysis of the differences among the independent research results, and the quantitative analysis and evaluation of the results of these statistical methods[2, 3]. Compared with traditional literature review, meta-analysis method has many advantages in literature review, such as enlarging sample number, improving statistical test efficiency and improving effect size estimation of research results[4, 5]. Since 1976, American educational psychologist Glass first formally proposed meta-analysis, the application of this method in the research of medicine, biology, psychology, education and other disciplines has increased rapidly[6]. Since 1989, meta-analysis has been applied in economic research, especially in the fields of environmental economics, labor economics, industrial organization theory and macroeconomics [5, 7]. The basic principles were being introduced in this articles and a brief literature review will be made in economic areas for providing evidence in practicing.

\section{What's meta-analysis and its basic steps}

Meta-analysis is widely used in evidence-based medicine, which may be regarded as a young statistical analysis that combines the results of multiple scientific studies[8]. However, the application of meta-analysis can be retrospective to 17 century used in astronomy. The great contribution was made by Glass in 1976 as the above mentioned, who stated that Meta-analysis refers to the analysis of analyses". Because of this, leading to him being widely recognized as the modern 
founder of the method, while the methodology behind what he termed "meta-analysis" predates his work by several decades[5].

The principles of meta-analysis are the same as the systematic review in accordance with the Preferred Reporting Items for Systematic Reviews and Meta-Analyses (PRISMA) guidelines[9], with no doubt. So in order to make a comprehensive analysis, based on general and systematic retrieval and collection of literature data, the collected literature should be scientifically and effectively sorted and classified. This is a basic task of the subsequent meta-analysis, and the following points should be noted.

The first stage, formulating a research question, based on the principles PICOs (population, intervention, control, outcomes, study design)[10]. Generally speaking, the questions to be studied in meta-analysis are those in the field to be studied where the conclusions are uncertain or controversial.

The second stage is to search for and collect literature and data related to the research problem. In meta-analysis, the comprehensive and systematic collection of literature materials and data related to the research problem is the basis for completing a high-quality meta-analysis report, and it is also one of the important features of meta-analysis different from the traditional literature review. On the basis of comprehensive and systematic retrieval and collection of literature data, it is necessary to scientifically and effectively sort and classify the collected literature. This is the basic work of the subsequent meta-analysis, and the following points should be noted. First, establish scientific and clear criteria for the inclusion and exclusion of literature. It is impossible for meta-analysis to analyze all the retrieved literature, but to select the qualified literature from the retrieved literature. This requires the development of criteria for the inclusion and exclusion of literature. Factors such as research objects, design types, treatment factors and outcome effects should be taken into account, as well as the selection of literature in terms of sample size, years of observation, time of publication and language. Secondly, the quality of the included literature was systematically evaluated. In this regard, the main work is to analyze whether there is bias in the research results of each literature (such as selective bias, publication bias, etc.) and the influence of such bias. The quality of each research result can be expressed by weight, or by scale or scoring system. Finally, the data information of the included literature was extracted. The data information adopted in meta-analysis generally includes basic information, research characteristics, result measurement, etc., and effect variables that need to be analyzed and evaluated are determined and selected. If necessary, find a way to obtain unpublished raw data from the original authors of the literature.

In the third stage, select the appropriate "effect value" and carry out the corresponding statistical test and analysis. In meta-analysis, in order to make the results of a single study comparable, it is necessary to combine the results of multiple independent studies into a single effect value, that is, a combined statistic to reflect the combined effect of multiple independent studies. Therefore, "effect size" is a core concept in meta-analysis. The scientificity and rationality of the results of a meta-analysis largely depend on whether the "effect value" selected is appropriate and scientific.

In the fourth stage, explain and draw your own conclusions. In essence, Meta-analysis is an observational research method, so we should be cautious about the interpretation of the results. When reporting meta-analysis results, discussion and analysis should also be carried out based on the research background and practical significance involved in the original literature. If necessary, the consistency between the results of a single study with a large sample and the results of meta-analysis can also be studied.

\section{The statistic model of meta-analysis}

Hedges' g, which is a popular summary measure for continuous data that is standardized in order to eliminate scale differences, but it incorporates an index of variation between groups.

$$
\delta=\frac{\mu_{t}-\mu_{c}}{\sigma} \text { which } \mu_{t} \text { is the treatment mean, and } \mu_{c} \text { is the control mean, } \sigma^{2} \text { represented the }
$$

pooled variance. 
For calculating the T-test, the formula shown at the below mentioned

$$
r=\frac{t}{\sqrt{t^{2}+d f}}
$$

For the bias of included studies, the Cochrane Collaboration's risk of bias tool is widely used for assessing the bias of RCTs[11]. This tool contains seven (7) items which are as follows: random sequence generation (selection bias), allocation concealment (selection bias), blinding of participants and personnel (performance bias), blinding of outcome assessment (detection bias), incomplete outcome data (attrition bias), selective reporting (reporting bias), and other bias.

\section{The application used in economics}

Over the years, the research methods of economics have been greatly improved, especially the rapid development of econometrics and experimental economics, and the meta-analysis provides a realistic basis for the application of meta-analysis to study economic problems. In fact, in recent years, meta-analysis has been widely applied and developed in many fields of economics.

Philipp et al 2020, has investigated that if economic globalization affects government spending, and they show further that several factors influence the globalization-spending estimates reported in the literature, including the choice of the economic globalization indicator, details of the econometric specifications, and publication characteristics [12]. Huang 2018 et al has study the effects of warming on agriculture from the econometric point, based on meta-analysis, and they found that the disagreement could be reduced if the studies account for adaptation by using a yearly temperature measure and the hedonic approach. [13]. Manzano 2019 et al has analyzed the complex relationships between increases to speed limits and traffic fatalities, which suggests by count traffic fatalities went up on both rural interstates and statewide level, although the effect was higher on rural interstates. In other respects, statewide fatality rates could be improved in relative terms by raising legal speed limits, although the effect would be weak[14].

As a means of economic analysis, Meta-analysis, although there are some limitations, such as primary literature publication bias, different sources of heterogeneity, but it provides a set of qualitative and quantitative analysis of combining the analysis framework and rules, and the results of objectivity and consistency of quality, there is no doubt that the prevalence of the same for the current economics research has multiple complex economic problems of the findings provide a comprehensive and scientific and reasonable evaluation has an important significance and function.

\section{Future implication}

We should believe that, with the vigorous development of the economics research in China, the emergence of a large quantity of economics research literature, is bound to need more scientific review like Meta-analysis tool for integrated solutions to a various economic problem, clarify the thinking of economic development, make our country a further improvement and development of the economics study.

\section{References}

[1] Stanley TD, Doucouliagos C, Jarrell SB. Meta-regression analysis as the socio-economics of economics research[J]. Journal of Socioeconomics. 2008,37(1):0-292.

[2] Joury E, Bernabe E, Sabbah W, Nakhleh K, Gurusamy K. Systematic review and meta-analysis of randomised controlled trials on the effectiveness of school-based dental screening versus no screening on improving oral health in children[J]. Journal of dentistry. 2017,58:1-10.

[3] Pillastrini P, Castellini G, Chiarotto A, Fasciani F, Marzioni F, Vanti C, et al. Comparative effectiveness of conservative and pharmacological interventions for chronic non-specific neck pain: Protocol of a systematic review and network meta-analysis[J]. Medicine. 2019,98(33):e16762. 
[4] Siddaway AP, Wood AM, Hedges LV. How to Do a Systematic Review: A Best Practice Guide for Conducting and Reporting Narrative Reviews, Meta-Analyses, and Meta-Syntheses[J]. Annual review of psychology. 2019,70:747-70.

[5] Alfirevic Z, Keeney E, Dowswell T, Welton NJ, Medley N, Dias S, et al. Methods to induce labour: a systematic review, network meta-analysis and cost-effectiveness analysis[J]. BJOG : an international journal of obstetrics and gynaecology. 2016,123(9):1462-70.

[6] Gurevitch J, Koricheva J, Nakagawa S, Stewart G. Meta-analysis and the science of research synthesis[J]. Nature. 2018,555(7695):175-82.

[7] Alfirevic Z, Keeney E, Dowswell T, Welton NJ, Medley N, Dias S, et al. Which method is best for the induction of labour? A systematic review, network meta-analysis and cost-effectiveness analysis[J]. Health technology assessment (Winchester, England). 2016,20(65):1-584.

[8] McCool R, Wilson K, Arber M, Fleetwood K, Toupin S, Thom H, et al. Systematic review and network meta-analysis comparing ocrelizumab with other treatments for relapsing multiple sclerosis[J]. Multiple sclerosis and related disorders. 2019,29:55-61.

[9] Moher D, Liberati A, Tetzlaff J, Altman DG. Preferred reporting items for systematic reviews and meta-analyses: the PRISMA statement[J]. Annals of internal medicine. 2009,151(4):264-9, w64.

[10] Eriksen MB, Frandsen TF. The impact of patient, intervention, comparison, outcome (PICO) as a search strategy tool on literature search quality: a systematic review[J]. Journal of the Medical Library Association : JMLA. 2018,106(4):420-31.

[11] Higgins JP, Altman DG, Gøtzsche PC, Jüni P, Moher D, Oxman AD, et al. The Cochrane Collaboration's tool for assessing risk of bias in randomised trials[J]. BMJ (Clinical research ed). 2011,343:d5928.

[12] Heimberger P. Does economic globalization affect government spending? A meta-analysis[J]. Public Choice.26.

[13] Huang KX, Sim N. Why do the econometric-based studies on the effect of warming on agriculture disagree? A meta-analysis[J]. Oxf Econ Pap-New Ser. 2018,70(2):392-416.

[14] Castillo-Manzano JI, Castro-Nuno M, Lopez-Valpuesta L, Vassallo FV. The complex relationship between increases to speed limits and traffic fatalities: Evidence from a meta-analysis[J]. Saf Sci. 2019,111:287-97. 